\title{
Salicylic acid, A Versatile Hormone to combat diseases in cotton
}

\author{
P. Valarmathi* \\ (Plant Pathology), ICAR-Central Institute for Cotton Research (CICR), Regional station, \\ Coimbatore, India \\ *Corresponding author
}

A B S T R A C T

\section{Keywords}

Salicylic acid, Signaling, metabolism, Systemic acquired resistance, Hypersensitive response, Hormonal cross talk, Cotton diseases

\section{Article Info}

Accepted:

05 June 2020

Available Online:

10 July 2020
The plant hormone salicylic acid (SA) plays an essential role in the regulation of diverse biological processes throughout the entire lifespan of the plant. SA first emerged as an endogenous signal capable of inducing plant defence responses both at the site of infection and in the systemic tissue of the plant. For more than 200 years, the plant hormone salicylic acid (SA) has been studied for its medicinal use in humans. In contrast, evident during the past 20 years has shown its extensive signaling role in plants particularly in defense against pathogens. The activation of Systemic Acquired Resistance (SAR) is associated with the discriminating level of expression of the pathogenesis-related proteins, which possess antimicrobial activity. We discuss the progress made in understanding SA biosynthesis and signaling, its relationship with other mechanisms in plant defense and the practical utility in targeting this defense mechanism for enhancing disease resistance in cotton.

\section{Introduction}

The host when recognized by the pathogenderived elicitors, a series of alarm signals are send out to host cell proteins and to nuclear genes which causes them to become activated, to produce substances inhibitory to the pathogen. Elicitors are signal molecules which when bound with binding sites in plasma membrane, three kinds of signals are produced for activation of defense genes:
Intracellular signals, short-distance intercellular signals and systemic signals. Systemic signal transduction, which leads to systemic acquired resistance, is thought to be carried out by salicylic acid (SA), oligogalacturonides released from plant cell walls, jasmonic acid, systemin, fatty acids, ethylene and others. Some natural or synthetic chemicals, such as salicylic acid and the synthetic dichloroisonicotinic acid, also activate the signaling pathway that leads to 
systemic acquired resistance against several diverse types of plant pathogenic viruses, bacteria and fungi.

Host recognition of pathogen elicitors initiates early signaling events such as protein phosphorylation/ dephosphorylation, ion fluxes and oxidative burst. Subsequent transcriptional and/or posttranslational activation of transcription factors leads to induction of plant defense genes and biosynthesis of endogenous secondary signals such as SA. In addition, the activated NADPH oxidase complex generates reactive oxygen species (ROS) such as $\mathrm{O}_{2}$ and $\mathrm{H}_{2} \mathrm{O}_{2}$ that alter the redox status of plant cells and affect defense signaling. SA, ROS, as well as defense genes, all contribute to the development of Hypersensitive Reaction and SAR during plant-pathogen interactions.

Salicylic acid (SA) plays an important role in plant defense. Its role in plant disease resistance is well documented for dicotyledonous plants, where it is required for basal resistance against pathogens as well as for the inducible defense mechanism, systemic acquired resistance (SAR), which confers resistance against a broad-spectrum of pathogens. Salicylic acid (SA) is an important plant hormone that regulates many aspects of plant growth and development, as well as resistance to (a)biotic stress. Studies in the model plant Arabidopsis thaliana has provided important insights into the mechanism of SA signaling in plant defense. The NPR1 protein is an important component of SA signaling in Arabidopsis. NPR1 is also required for plant defense mechanisms that do not require SA. Hence, NPR1 provides an important link between different defense mechanisms. Similarly, cross talk between SA and other defense signaling pathways results in the fine-tuning of plant defense response. This review depicts how discovery of SA occurs and its role in plants as signal molecules which induces both local disease resistance mechanisms, including host cell death and defense gene expression and systemic acquired resistance (SAR).

\section{Discovery of salicylic acid}

Salicylic acid (SA) is a molecule which produces signal naturally found in plants and shown to be involved in the plant defenserelated actions against infection by various pathogens. SA biology has a long history, dating back to the ancient time, very much prior to application of SA for combating the plant diseases. Plant crude extracts which contains phenolics such as SA, saligenin and its glucoside salicin have been used as sorts of medicinal agents against humans. Over centuries until $18^{\text {th }}$ century, the name of SA and related compounds originally came from the Salix helix (willow) tree, since they were discovered as the major components in the extracts from willow tree bark or poplar tree bark that had been used as natural antiinflammatory drugs (Weissman, 1991). In 1897, Bayer company produced acetylsalicylic acid which is widely known as aspirin as anti-inflammatory agent by mimicking the action of the ancient medicine from the willow tree. This is the world first synthetic drug to be known (Weissman, 1991). Studies relating salicyclates with plant disease resistance was initiated in early 1970's since the application of aspirin against a plant virus in growing leaves was shown to be effective (White, 1979). Antoniw and White, 1980 were the first plant biologists whom they paid attention to salicyclates as disease resistance-inducing chemicals. They both demonstrated that injection of aspirin into tobacco leaves enhanced the resistance to subsequent infection by Tobacco Mosaic Virus (TMV). Subsequent studies have shown that aspirin and SA induce the accumulation of pathogenesis-related (PR) proteins (Kessman and Ryals, 1993). 
Plants rich in SA and its derivatives, collectively termed salicylates, have been used for medicinal purposes for millennia. In the fourth century BC, willow leaves/bark extracts were prescribed by Hippocrates to relieve fever and the pain of childbirth (Vlot et al., 2009). Salicylate-rich plants were also used by other ancient cultures, including the Babylonians, Assyrians and Chinese, as well as the indigenous inhabitants of the New World (Kahn et al., 2015). In the mid-1700s by the Reverend Edward Stone, effects were first studied clinically for willow bark was a well-known folk remedy (Weissman, 1991). In 1828, Johann Buchner purified the active ingredient in willow bark and named it salicin. Raffaele Piria subsequently demonstrated that salicin could be split into a sugar and an aromatic compound he named SA, in reference to Salix alba, the Latin name for white willow (Klessing et al., 2016). Around this time, high levels of salicylates were detected in other medicinal plants, such as meadowsweet, which contains both salicin and MeS- then called oil of wintergreen (Weissman, 1991; Klessing et al., 2016). These "prodrugs" are converted to SA after digestion in humans/ animals. Increased demand for SA in the mid-1800s led to the commercial production of synthetic SA in 1874. As SA's price fell and its availability increased, its clinical use expanded. However, SA's negative side effects, particularly stomach irritation, precluded long-term, highdosage use. Research by Felix Hoffmann revealed that acetylation improved SA's tolerability without affecting its medicinal properties (Weissman, 1991). Bayer Company began synthesizing acetyl SA (ASA) in 1897 under the trade name aspirin, which was generated by combining the "a" from acetyl and "spirin" from the Latin name for meadowsweet (Spiraea ulmaria). Today, aspirin is one of the most widely used drugs in the world. In addition to treating fever, swelling, pain, and inflammation, aspirin is used prophylactically to reduce the risk of stroke, heart attack, and certain cancers (Weissman, 1991; Cuzick et al., 2015).

\section{Salicylic acid as a key immune hormone in plants}

Salicylic acid (SA) is an important secondary phenolic metabolite in a wide range of prokaryotic and eukaryotic organisms, including plants. It is well established that SA plays crucial roles in diverse biological processes, such as plant cell growth, seed germination and development, thermotolerance, respiration, stomatal aperture, fruit yield, nodulation in legumes and leaf senescence (Fig. 1). More importantly, it serves as a key signalling and regulatory molecule in plant defence responses and has thus come to be regarded as the key plant immune hormone. Upon recognition of a pathogen, the cellular concentrations of SA and pathogenesisrelated (PR) proteins become elevated. In addition to the localized immune responses at the site of infection, SA is capable of inducing a heightened state of resistance in distal tissue. This state of increased preparedness for a potential infection throughout the plant is known as systemic acquired resistance (SAR) (Liu et al., 2015).

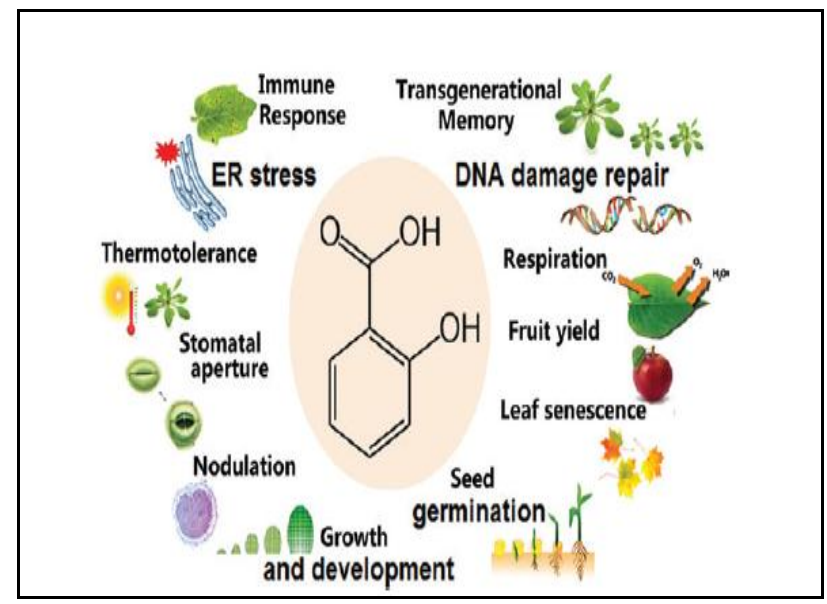

Figure.1 Biological functions of salicylic acid in plants (Derived from Liu et al., 2015). 
Salicylic acid (SA) regulates a multitude of developmental processes and stress responses in plants. Along with the well-established functions of SA in plant immune responses, plant cell growth, seed germination, thermotolerance, respiration, stomatal aperture, fruit yield, nodulation and leaf senescence, novel functions are coming to light, such as roles in ER stress, DNA damage repair and transgenerational memory, among others.

\section{Salicylic acid as a plant hormones/ signal molecule}

The first confirmation that SA is a plant hormone came from studies of voodoo lily (Sauromatum guttatum Schott) (Raskin, 1992). During blooming, the voodoo lily inflorescence exhibits two episodes of thermogenesis (heat production). These two events increase the surface temperature of the inflorescence by 12 and $10{ }^{\circ} \mathrm{C}$ and are thought to volatilize compounds that attract/stimulate insect pollinators. Internal SA levels increased $\sim 100$-fold prior to each episode (Raskin, 1992). Externally supplied SA and two closely related analogs also induced thermogenesis, whereas 31 other SA analogs did not induce. SA induces thermogenesis primarily by stimulating the mitochondrial alternative respiratory pathway (Rhoads et al., 1992). This pathway, unlike the cytochrome respiratory pathway, generates ATP at just one step and releases the remaining energy from electron flow as heat. Interestingly, SA treatment also induces the expression of alternative oxidase and/or the alternative respiratory pathway in non-thermogenic plant species (Norman et al., 2004; Clifton et al., 2005). The year after SA's function in thermogenesis was elucidated, its role as a defense signaling hormone was documented. SA also has long been proposed as a signal for flowering. Interestingly, several proteins that regulate both flowering and resistance signaling have been identified (Jin et al., 2008; Li et al., 2012; Fortuna et al., 2015; Wang et al., 2011; Liu et al., 2014; Lee et al., 2006; Tsuchiya et al., 2010 and Singh et al., 2013). While this finding suggests an interconnection between flowering and disease resistance (Banday et al., 2015), it is clearly complex: some of these proteins regulate both resistance and flowering in an SA-dependent manner, whereas several others regulate resistance via an SA-dependent pathway but positively or negatively regulate flowering via an SA-independent mechanism.

In view of the fact that the late 1970s, it was known that applying SA to tobacco plants induces defense gene expression and enhances resistance to viral infection (White et al., 1979). However, SA's role as an internal signal for disease resistance was not demonstrated until 1990, when rises in SA levels were detected prior to the development of local and/or systemic disease resistance in tobacco and cucumber (Malamy et al., 1990; Metraux et al., 1990). Analyses of tobacco and Arabidopsis unable to accumulate SA (due to various mutations or expression of SA-degrading enzymes) confirmed that SA is required for PTI, ETI, and SAR (Vlot et al., 2009). Grafting studies using SA-deficient or wild-type (wt) tobacco further indicated that while SA accumulation is required in the uninfected leaves for SAR development, SA is not the mobile SAR-inducing signal that travels from the inoculated to systemic leaves (Vernooij et al., 1994; Pallas et al., 1996). SA's role as a defense signal has been extended to many plant species. However, there are conflicting reports regarding its role in some monocots (Vlot et al., 1990) as well as in plants that constitutively accumulate high levels of SA (such as potato and rice); its role in some plant species also appears to vary depending on the pathogen (Brading et al., 2000; Sánchez et al., 2010). It should be noted that SA is one of several plant 
hormones involved in signaling defenses against microbial pathogens (RobertSeilaniantz et al., 2011). The SA-mediated defense signaling pathway is activated following infection by biotrophic pathogens, which require living host tissue. By contrast, attack by necrotrophic pathogens, which feed on dead tissue, induces a distinct defense pathway that is regulated by the plant hormones jasmonic acid (JA) and ethylene. The SA- and JA/ethylene-mediated defense pathways undergo extensive cross-talk; their interactions are generally antagonistic (De Vleesschauwer et al., 2014).

\section{Biosynthesis of salicylic acid in plants}

The two major SA biosynthetic pathways in plants were identified as: the isochorismate (IC) and the phenyl alanine ammonia-lyase (PAL) pathways. Both pathways commonly utilize chorismate, the end product of the shikimate pathway, to produce SA (Dempsey et al., 2011). IC synthase (ICS) and PAL are critical enzymes for these pathways, respectively. Homologs of ICS and PAL genes are present throughout the plant kingdom, including Arabidopsis, tobacco, tomato, populus, sunflower and pepper (Wildermuth et al., 2001; Cochrane et al., 2004; Uppalapati et al., 2007; Catinot et al., 2008; Yuan et al., 2009; Sadeghi et al., 2013; Kim and Hwang, 2014), suggesting the importance of these SA biosynthesis pathways to survive during the course of evolution. In Arabidopsis, mutations in ICS1 lead to an almost complete loss of pathogeninduced SA accumulation (Wildermuth et al., 2001). However, Arabidopsis quadruple PAL mutants, in which PAL activity is reduced to $10 \%$, also show lower SA accumulation (50\%) compared to the wild type upon pathogen infection (Huang et al., 2010). Thus, while contribution of the PAL pathway is evident, the IC pathway is the major route for SA biosynthesis during plant immunity. In chloroplasts, ICS catalyzes the conversion of chorismate into IC (Wildermuth et al., 2001; Strawn et al., 2007; Garcion et al., 2008), which is further converted to SA (Dempsey et al., 2011). In some bacteria, conversion of IC to SA is catalyzed by IC pyruvate lyases (IPLs; Dempsey et al., 2011). However, plant genomes encode no homologous genes to bacterial IPLs. Expression of bacterial enzymes catalyzing this conversion together with ICS in chloroplasts leads to constitutive accumulation of SA (Verberne et al., 2000; Mauch et al., 2001). Salicylic acid biosynthesis is tightly regulated since constitutive SA accumulation has negative impacts on plant fitness (Pajerowska-Mukhtar et al., 2012; Chandran et al., 2014). Accumulating evidence show that transcriptional control of ICSI by calcium signaling is key for the initiation of SA biosynthesis. Li et al., 2018 studied SA in Populus tomentosa infected by the plant pathogen Botryosphaeria dothidea

\section{Resistance of plants to pathogen infection}

Eventhough plants lack the circulating immune cells found in vertebrates, they do possess an innate immune system that detects and limits pathogen colonization (Thomma et al., 2011; Spoel and Dong, 2012 and Asai et al., 2015). One branch of this system uses pattern recognition receptors (PRRs) on the plant cell surface to survey for molecules containing characteristic patterns that are unique to and broadly conserved in microbes. Detection of these pathogen- $/$ microbeassociated molecular patterns (PAMPs/ MAMPs) leads to activation of patterntriggered immunity (PTI). In many cases, PTI prevents further pathogen colonization. However, some pathogens have evolved effector proteins that suppress PTI. These pathogens are combatted via effectortriggered immunity (ETI), which comprises the other branch of the innate immune system. ETI is activated when plant-encoded resistance (R) proteins, which are generally 
located within the plant cell, directly or indirectly recognize their cognate pathogen encoded effectors. Both PTI and ETI are associated with the activation of defenses in the inoculated tissue, including the generation of reactive oxygen species (ROS), increases in intracellular $\mathrm{Ca}^{2+}$ concentrations, activation of mitogen activated protein kinases (MAPKs), increased expression of various defense-associated genes, synthesis of antimicrobial compounds and accumulation of SA (Seyfferth et al., 2014; Stael et al., 2015). Generally, ETI induces these defenses more rapidly and intensely than PTI. ETI also is usually associated with necrotic lesion formation, which may help restrict pathogen movement from the infection site. Subsequent to these events, ETI and PTI can induce immune responses in the uninoculated (systemic) portions of the plant, including a long-lasting, broad-spectrum resistance called systemic acquired resistance (SAR) (Dempsey and Klessig, 2017).

\section{Systemic acquired resistance (SAR)}

Systemic resistance is induced by pathogens and confers protection against a broad range of pathogens. Studies have indicated that salicylic acid (SA) derivative methyl salicylate (MeSA) serves as a long-distance phloem-mobile systemic resistance signal in tobacco, Arabidopsis, and potato. However, other experiments indicate that jasmonic acid (JA) is a critical mobile signal. Evidence suggesting both MeSA and methyl jasmonate (MeJA) are essential for systemic resistance against Tobacco mosaic virus (TMV), possibly acting as the initiating signals for systemic resistance (Zhu et al., 2014). Foliar application of JA followed by SA triggered the strongest systemic resistance against TMV.

Systemic acquired resistance (SAR) is a mechanism of induced defense which confers long-lasting protection against a broad spectrum of microorganisms. SAR requires the signal molecule salicylic acid (SA) and that is associated with accumulation of pathogenesis-related proteins (PR), which are thought to contribute to resistance. Much progress has been made recently in elucidating the mechanism of SAR. Using the model plant Arabidopsis, it was discovered that the isochorismate pathway is the major source of SA during SAR. In response to SA, the positive regulator protein NPR1 moves to the nucleus where it interacts with TGA transcription factors to induce defense gene expression, thus activating SAR (Chaturvedi and Shah, 2007). Durrant and Dong, 2004 discussed in detail the molecular and genetic data which have contributed to the understanding of SAR and present a model describing the sequence of events leading from initial infection to the induction of defense genes. SA accumulation is essential for expression of multiple modes of plant disease resistance. In higher plants, salicylic acid (SA) plays important roles in inducing resistance to biotic and abiotic stresses. Tomato yellow leaf curl virus (TYLCV) causes a highly devastating viral disease in plants, particularly in tomato ( $\mathrm{Li}$ et al., $2019 b$ ). The activation of SAR provides a broad-spectrum resistance against a wide range of related or unrelated pathogens. There has been considerable progress in the biochemical and molecular understanding of SAR activation in various plants (Tripathi et al., 2019).

Systemic acquired resistance (SAR) is a secondary immune response in the distal parts of plants activated by local defense responses (Fig. 2). SAR is long-lasting and effective against a broad spectrum of pathogens, including fungi, bacteria, and viruses. Traditionally, SAR is induced by incompatible pathogens that cause localized cell death. Tissue necrosis at inoculation sites 
is not required for SAR activation, however. Salicylic acid (SA) is a phytohormone that plays a central role in defense signaling (Vlot et al., 2009). It is required for both basal defense and SAR. Early studies showed that pathogen infections lead to increased SA levels in both local and distal parts of plants (Malamy et al., 1990; Metraux et al., 1990). Whereas application of exogenous SA or SA analogs induces resistance to pathogens, degradation of SA by transforming plants with the bacterial salicylate hydroxylase gene NahG blocks SA accumulation and SAR (Gaffney, 2013).

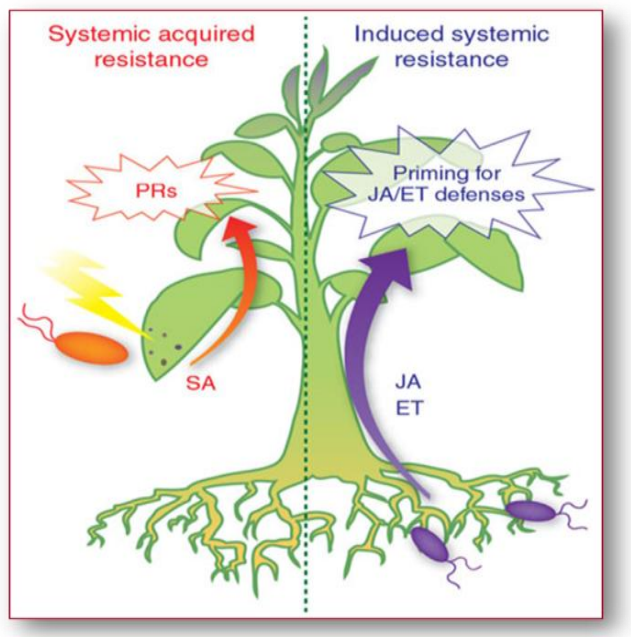

Fig.2 Role of Salicylic Acid and Jasmonic Acid as systemic signals (Derived from Zhu et al., 2014)

\section{SA research benefitting agriculture}

Synthetic pesticides have allowed growers to dramatically increase crop yield and quality. However, these compounds are often toxic and their overuse has led to development of resistance in pathogen (Schreinemachers and Tipraqsa, 2012; Lucas et al., 2015). An environmentally friendly strategy for reducing crop loss involves regulating SAR (da Rocha and Hammerschmidt, 2005). SA is an effective SAR inducer, but its phytotoxicity precludes widespread use (Conrath et al., 2015). Several synthetic compounds, including 2,6- dichloroisonicotinic acid (INA), benzo(1,2,3)thiadiazole-7-carbothioic acid S-methyl ester (also termed benzothiadiazole; BTH or acibenzolar- Smethyl), and probenazole (PBZ) and its active metabolite 1,2-benzisothiazol-3(2H)-one 1,1dioxide (BIT), induce defense gene expression and SAR to a similar range of pathogens as SA (Gozzo and Faoro, 2013; Walters et al., 2013). PBZ and BIT activate SAR by triggering the SA signaling pathway upstream of SA (Yoshioka et al., 2001) whereas INA and BTH are SA functional analogs (Vallad and Goodman, 2004). While treating plants or suspension cells with high concentrations of SA or its functional analogs directly induces defenses, low concentrations elicit little to no response. Following subsequent infection, however, defenses are activated more rapidly and/or strongly (Conrath et al., 2006). This phenomenon, termed priming, also occurs in systemic leaves of plants exhibiting SAR. Although not fully elucidated, the molecular mechanisms of priming likely involve the accumulation of transcripts and/or inactive forms of MAPKs, elevated levels of PRRs, and chromatin remodeling. This latter mechanism may also promote the inheritance of defense priming. In addition to SA, its functional analogs and PBZ/BIT, other resistance-activating compounds that work at least in part by inducing/priming SAR, have been identified (Choi and Hwang, 2001). In the field, resistance triggered by SAR inducers/primers can be broad-spectrum and long-lasting, but it is rarely complete; disease reduction ranges from $20-85 \%$. Other drawbacks growers must currently consider include i) the potential for reduced plant fitness, which may be minimized by stimulating priming versus direct induction (van Hulten et al., 2006) ii) variable efficacy, depending on plant cultivar and dosage, and iii) the possibility that SAR inducers will increase susceptibility to necrotrophic pathogens, due to suppression of 
the JA signaling pathway (Gozzo and Faoro, 2013; Walters et al., 2013; Guo et al., 2019).

\section{Developing better defensive plants via deciphering crosstalk}

Plant defense responses against environmental pathogens seems to be energy consuming one. Ideally, plants employ a specific pathway upon recognizing of distinct pathogens. Extensive crosstalk between different signaling pathways provides the potential for efficient energy allocation. For instance, the SA and ET/JA mediated defense signaling pathways act in both synergistically and antagonistically. Treatment with low concentrations of SA and JA has been reported to result in synergistic expression of both the SA target gene PR1 and the JA marker gene PDF1.2, whereas higher concentrations of SA and JA produce the antagonistic expression of these genes (Mur et al., 2006). So far, the metabolism and signaling transduction of SA, ET and JA have been well elucidated. But, do we fully understand the signaling crosstalk between those hormones? Probably not. In nature, defense hormones work together to manage invading pathogens in an ecological context. Due to the high level of complexity, the mechanism that underlies the crosstalk is poorly understood and requires further study. Here envision that the newly emerged largescale OMIC tools and high throughput bioinformatic analysis will be used to seek a better understanding of the crosstalk between these defense hormones, which will ultimately lead to the development of pathogen-resilient crop plants with important agronomical perspectives.

For instance, if the crosstalk between SA and ET/JA-signaling pathways is disconnected, plants will be able to defend against simultaneously colonized biotrophic and necrotrophic pathogens without tradeoffs.
Under such conditions, both SA- and ET/JAsignaling pathways are fully armed to fight against corresponding enemies. As the connection node that mediates the crosstalk is still unknown, the gene-piling CRISPR/cas technology serves no purpose. It would be fascinating if, by genetic engineering, the SAmediated signaling pathway could be rewired to control the ET/JA-signaling pathway. Early biotroph infection will prime the plants for potential necrotrophs in the environment. Interestingly, the SA receptor NPR3/4 has been shown to activate the JA-signaling pathway by promoting the degradation of the JA transcriptional repressor JAZs (Liu et al., 2016).

Both positive and negative regulatory factors of the signaling pathways are probable targets to modulate defense hormonal crosstalk. It is a particularly exciting area of study to address the signaling crosstalk between those defense hormones, which bears the promise of developing better plants. The ET/JA-mediated response contributes to the defense against necrotrophic pathogens, such as Botrytis cinerea, which invade and kill hosts to extract their nutrients. Increasing evidence indicates that the SA- and ET/JA-mediated defense response pathways are mutually antagonistic.

Salicylic acid-related cotton (Gossypium arboreum) ribosomal protein GaRPL18 contributes to resistance to Verticillium dahliae

Verticillium dahliae is a phytopathogenic fungal pathogen that causes vascular wilt diseases responsible for considerable decreases in cotton yield. Gong et al., 2017 cloned the ribosomal protein L18 (GaRPL18) gene, which mediates resistance to Verticillium wilt, from a wilt-resistant cotton species (Gossypium arboreum). They characterized the function of this gene in cotton and Arabidopsis thaliana plants. 
GaRPL18 encodes a $60 \mathrm{~S}$ ribosomal protein subunit important for intracellular protein biosynthesis. However, the earlier studies revealed that some ribosomal proteins are also inhibitory towards oncogenesis and congenital diseases in humans and play a role in plant disease defense. Here, they observed that $V$. dahliae infections induce GaRPL18 expression. Furthermore, they determined that the GaRPL18 expression pattern is consistent with the disease resistance level of different cotton varieties. GaRPL18 expression is upregulated by salicylic acid (SA) treatments, suggesting the involvement of GaRPL18 in the SA signal transduction pathway.

Virus-induced gene silencing technology was used to determine whether the GaRPL18 expression level influences cotton disease resistance. Wilt-resistant cotton species in which GaRPL18 was silenced became more susceptible to $V$. dahliae than the control plants because of a significant decrease in the abundance of immune-related molecules. They also transformed A. thaliana ecotype Columbia (Col-0) plants with GaRPL18 according to the floral dip method. The plants overexpressing GaRPL18 were more resistant to $V$. dahliae infections than the wild-type Col-0 plants. The enhanced resistance of transgenic A. thaliana plants to $V$. dahliae is likely mediated by the SA pathway. The findings provide new insights into the role of GaRPL18, indicating that it plays a crucial role in resistance to cotton "cancer", also known as Verticillium wilt, mainly regulated by an SA-related signaling pathway mechanism. This study is the first to examine the ribosomal proteins function related to cotton resistance to $V$. dahliae. Finally, this study revealed that SA is an important factor related to the cotton defense response system and that the mechanism of GaRPL18associated $V$. dahliae resistance is related to the SA signaling pathway.
Gbvdr6, a gene encoding a receptor-like protein of cotton (Gossypium barbadense), confers resistance to Verticillium wilt in Arabidopsis and upland cotton

Verticillium wilt is a soil-borne disease that can cause devastating losses in cotton production. Because there is no effective chemical means to combat the disease, the only effective way to control Verticillium wilt is through genetic improvement. Therefore, the identification of additional diseaseresistance genes will benefit efforts toward the genetic improvement of cotton resistance to Verticillium wilt. Based on screening of a BAC library with a partial $V e$ homologous fragment and expression analysis, a $V$. dahliae-induced gene, Gbvdr6, was isolated and cloned from the Verticillium wilt-resistant cotton G. barbadense cultivar Hai7124. The gene was located in the gene cluster containing Gbvel and Gbvdr5 and adjacent to the Verticillium wilt-resistance QTL hotspot. Gbvdr6 was induced by Verticillium dahliae Kleb and by the plant hormones salicylic acid (SA), methyl jasmonate (MeJA) and ethephon (ETH) but not by abscisic acid (ABA). Gbvdr6 was localized to the plasma membrane.

Over expression of Gbvdr6 in Arabidopsis and cotton enhanced resistance to $V$. dahliae. Moreover, the JA/ET signaling pathwayrelated genes $P R 3, P D F$ 1.2, ERF1 and the SA-related genes $P R 1$ and $P R 2$ were constitutively expressed in transgenic plants. Gbvdr6-overexpressing Arabidopsis was less sensitive than the wild-type plant to MeJA. Furthermore, the accumulation of reactive oxygen species and callose was triggered at early time points after $V$. dahliae infection. These results suggest that Gbvdr6 confers resistance to $V$. dahliae through regulation of the JA/ET and SA signaling pathways (Yang et al., 2017). 
Systemic induced resistance to Alternaria macrospora in cotton (Gossypium hirsutum)

Inoculation of cotyledons with Alternaria macrospora or application of 2, 6dichloroisonicotinic acid to cotyledons caused the next emerging leaves to become less susceptible to infection by $A$. macrospora than those on control plants. This effect was demonstrated in cotton cultivars Siokra and Deltapine using young plants raised and tested under glasshouse conditions.

The treatments applied to the cotyledons had no visible effect on the development of the plants and 2,6-dichloroisonicotinic acid had no direct effect on the fungus in vitro, suggesting that resistance was induced systemically in cotton plants (Brock et al., 1994).

\section{Salicylic acid act as a signal in cotton for induced resistance to Helicoverpa zea}

Bi et al., 1997 indicated that insect herbivory on cotton induced resistance to the cotton bollworm (Helicoverpa zea). They examined the role of salicylic acid as a signal in cotton for the induced resistance. Abundant evidence has accumulated showing that salicylic acid plays a key role in coordinating the expression of systemic acquired resistance against phyto-pathogens. They reported that herbivory results in significant increases in foliar salicylic acid and $\mathrm{H}_{2} \mathrm{O}_{2}$, a response frequently observed following pathogenesis. In other well-studied systems (e.g., tobacco), salicylic acid inhibits the enzymatic decomposition of $\mathrm{H}_{2} \mathrm{O}_{2}$ by catalase and ascorbate peroxidase, but in cotton, salicylic acid has no effect on these enzymes in vitro. Furthermore, while herbivory enhances foliar catalase and ascorbate peroxidase activities, the application of salicylic acid or methyl salicylate to cotton plants does not affect foliar resistance to $H$. zea.

\section{Concluding remarks with future thrusts}

Over the last 25 years, considerable progress has been made in the identification and characterization of a number of salicylic acid signalling components that are implicated in a myriad of cellular pathways. Despite significant progress in elucidating the SA signaling pathway for plant disease resistance, significant knowledge gaps remain. Our understanding of the mechanism(s) through which pathogen perception is transduced by PRRs and $R$ proteins into activation of early cellular responses and SA synthesis is fragmentary. Likewise, the enzymes involved in SA biosynthesis are not fully identified and the role of the ICS versus PAL pathways in different plant species remains unclear. The mechanisms through which ICS1 expression is regulated, both in the nucleus and via retrograde signals from the chloroplast, also need to be determined. As for the signaling pathway downstream of SA, a crucial line of study will involve identifying SA targets/receptors and assessing their function. These analyses, combined with the development of in vivo SA detection techniques, should provide tremendous insight into how SA exerts its myriad effects. Elucidation of the SA signaling pathway should benefit agriculture by suggesting strategies for improving current SARinducing compounds, as well as facilitating development of novel compounds that target currently unidentified pathway components. Even more importantly, such future discoveries may also provide promising targets for marker-assisted selection and breeding by design in crops, thus promoting the sustainability of modern agriculture. Thus Salicylic acid (SA) acts as an important signalling role in the activation of plant defence responses following pathogenic invasion, including both systemic and localized responses usually characterized by HR. SA meets the essential criteria of a signal 
molecule, namely: (a) SA induces resistance to pathogens; (b) SA induces PR proteins; (c) SA levels increase locally and systemically following pathogen attack; and (d) SA moves throughout the plant via phloem. Nevertheless, future efforts to clarify the SA signaling pathway should provide insights into how current SAR-inducing compounds can be improved, as well as to identify novel pathway components that could be targeted by the next generation of agrochemicals.

\section{References}

Antoniw L.F. and White R.P. (1980). The effects of aspirin and polyacrylicacid on soluble leaf proteins and resistance to virus infection in five cultivars of tobacco. Phytopatho. Z., 98: 331-341.

Asai S. and Shirasu K. (2015). Plant cells under siege: plant immune system versus pathogen effectors. Curr. Opin. Plant. Biol. 28:1-8.

Banday Z.Z. and Nandi A.K. (2015). Interconnection between flowering time control and activation of systemic acquired resistance. Front Plant Sci., 6:174. doi:10.3389/fpls.2015.00174.

Bi J.L., Murphy J.B. and Felton G.W. Does Salicylic Acid Act as a Signal in Cotton for Induced Resistance to Helicoverpa zea?. J. Chem. Ecol., 23, 1805-1818. https://doi.org/10.1023/B:JOEC.0000006 452.81324.b8

Brading P.A., Hammond-Kosack K.E., Parr A. and Jones J.D.G. (2000). Salicylic acid is not required for Cf-2- and Cf-9-dependent resistance of tomato to Cladosporium fulvum. Plant J., 23:305-18.

Brock P.M., Inwood J.R.B. and Deverall, B.J. Systemic induced resistance to Alternaria macrospora in cotton (Gossypium hirsutum). Austral. Plant Pathol., 23, 8185. https://doi.org/10.1071/APP9940081

Catinot J., Buchala A., Abou-Mansour E. and Metraux J.P. (2008). Salicylic acid production in response to biotic and abiotic stress depends on isochorismate in
Nicotiana benthamiana. FEBS Lett., 582: 473-478.doi:

10.1016/j.febslet.2007.12.039

Chandran D., Rickert J., Huang Y., Steinwand M.A., Marr S.K. and Wildermuth M.C. (2014). A typical E2F transcriptional repressor DEL1 acts at the Intersection of plant growth and immunity by controlling the hormone salicylic acid. Cell. Host. Microbe., 15: 506513.doi:10.1016/j.chom.2014.03.007.

Chaturvedi R. and Shah J. (2007) Salicylic Acid in Plant Disease Resistance. In: Hayat S., Ahmad A. (eds) Salicylic Acid: A Plant Hormone. Springer, Dordrecht. 335-370.

Choi H.W. and Hwang B.K. (2011). Systemic acquired resistance of pepper to microbial pathogens. J. Phytopathol., 159:393-400.

Clifton R., Lister R., Parker K.L., Sappl P.G., Elhafez D., Millar A.H., Day D.A. and Whelan J. (2005). Stress-induced coexpression of alternative respiratory chain components in Arabidopsis thaliana. Plant Mol Biol., 58:193-212.

Cochrane F.C, Davin L.B. and Lewis N.G.(2004).The Arabidopsis phenylalanine ammonia lyase gene family: kinetic characterization of the four PAL isoforms. Phytochemistry., 65: $1557-$

1564.doi:10.1016/j.phytochem.05.006

Conrath U., Beckers G.J.M., Flors V., GarcíaAgustín P., Jakab G., Mauch F., Newman M.A., Pieterse C.M.J., Poinssot B., Pozo M.J., Pugin A., Schaffrath U., Ton J., Wendehenne D., Zimmerli L. and MauchMani B. (2006). Priming: getting ready for battle. Mol. Plant. Microbe. Interact., 19:1062-71.

Conrath U., Beckers G.J.M., Langenbach C.J.G. and Jaskiewicz M.R. (2015). Priming for enhanced defense. Annu. Rev. Phytopathol., 53:97-119.

Cuzick J., Thorat M.A., Bosetti C., Brown P.H., Burn J., Cook N.R., Ford L.G., Jacobs E.J., Jankowski J.A., LaVecchia C., Law M., Meyskens F., Rothwell P.M., Senn H.J. and Umar A. (2015). Estimates of benefits and harms of prophylactic use of 
aspirin in the general population. Ann. Oncol., 26:47-57.

da Rocha A.B. and Hammerschmidt R. (2005) .History and perspectives on the use of disease resistance inducers in horticultural crops. Hort. technology., 15:518-29.

De Vleesschauwer D., Xu J. and Höfte M. (2014). Making sense of hormonemediated defense networking: from rice to Arabidopsis. Front. Plant. Sci., 5:611. doi:10.3389/fpls.2014.00611.

Dempsey A.D. and Klessig, D.F. (2017). How does the multifaceted plant hormone salicylic acid combat disease in plants and are similar mechanisms utilized in humans? BMC Biology, 15:23.

Dempsey D.A., Vlot A.C., Wildermuth M.C. and Klessig D.F. (2011). Salicylic Acid biosynthesis and metabolism. Arabidopsis Book., 9: e0156.doi: 10.1199/tab.0156

Durrant W.E. and Dong X. (2004). Systemic Acquired Resistance. Annu. Rev. Phytopathol., 42:185-209.

Fortuna A., Lee J., Ung H., Chin K., Moeder W. and Yoshioka K. (2015). Crossroads of stress responses, development and flowering regulation-the multiple roles of Cyclic Nucleotide Gated Ion Channel 2. Plant Signal Behav., 10:e989758.

Gaffney. T. (1993) Requirement of salicylic acid for the induction of systemic acquired resistance. Science., 261:754756.

Gao Q.M., Zhu S., Kachroo P. and Kachroo A. (2015). Signal regulators of systemic acquired resistance. Front Plant Sci., 6:228. doi:10.3389/fpls.2015.00228.

Garcion C., Lohmann A., Lamodiere E., Catinot J., Buchala A. and Doermann,P (2008). Characterization and biological function of the ISOCHORISMATE SYNTHASE 2 gene of Arabidopsis. Plant Physiol., 147: 1279-1287.doi: 10.1104/pp.108.119420

Gon Q., Yang Z., Wang X., Butt H, I., Chen, E., He S., Zhang C., Zhang X. and Li F. (2017). Salicylic acid-related cotton (Gossypium arboreum) ribosomal protein GaRPL18 contributes to resistance to Verticillium dahliae. BMC Plant Biol.,
17:59. doi: 10.1186/s12870-017-1007-5

Gozzo F. and Faoro F. (2013). Systemic acquired resistance (50 years after discovery): moving from the lab to the field. J. Agric. Food. Chem., 61:1247391.

Guo B., Liu C., Liang Y., Li N. and Fu Q. (2019). Salicylic Acid Signals Plant Defence against Cadmium Toxicity. Int. J. Mol. Sci., 20: 2960.

Huang J.,Gu M.,Lai Z.,Fan B., Shi K., Zhou Y. H. (2010). Functional analysis of the Arabidopsis PAL gene family in plant growth, development, and response to environmental stress. Plant Physiol., 153: 1526-1538.doi: 10.1104/pp.110.157370

Jin J.B., Jin Y.H., Lee J., Miura K., Yoo C.Y., Kim W.Y., Van Oosten M., Hyun Y., Somers D.E., Lee I., Yun D.J., Bressan R.A. and Hasegawa P.M. (2008). The SUMO E3 ligase, AtSIZ1, regulates flowering by controlling a salicylic acidmediated floral promotion pathway and through affects on FLC chromatin structure. Plant J., 53:530-40.

Kahn M.I.R., Fatma M., Per T.S., Anjum N.A. and Kahn N.A. (2015). Salicylic acidinduced abiotic stress tolerance and underlying mechanisms in plants. Front Plant Sci., 6:462. doi:10.3389/fpls.2015.00462.

Kessmann H. and Ryals J. (1993). Requirement of salicylic acid for the induction of systemic acquired resistance. Science., 261:754-756.

Kim D.S. and Hwang B.K. (2014).An important role of the pepper phenylalanine ammonia-lyase gene(PAL1) in salicylic acid dependent signalling of the defence response to microbial pathogens. $J$. Exp.Bot., 65: 2295-2306.doi: 10.1093/jxb/eru109

Klessig D.F., Tian M. and Choi H.W. (2016). Multiple targets of salicylic acid and its derivatives in plants and animals. Front Immunol., $\quad 7: \quad 206$ doi:10.3389/fimmu.00206.

Lee J., Nam J., Park H.C., Na G., Miura K., Jin 
J.B., Yoo C.Y., Baek D., Kim D.H., Jeong J.C., Kim D., Lee S.Y., Salt D.E., Mengiste T., Gong Q., Ma S., Bohnert H.J., Kwak S.S., Bressan R.A., Hasegawa P.M. and Yun D.J. (2006). Salicylic acidmediated innate immunity in Arabidopsis is regulated by SIZ1 SUMO E3 ligase. Plant J., 49:79-90.

Li W., Ahn I.P., Ning Y., Park C.H., Zeng L., Whitehill J.G.A., Lu H., Zhao Q., Ding B., Xie Q., Zhou J.M., Dai L. and Wang G.L. (2012). The U-box/ARM E3 ligase PUB13 regulates cell death, defense and flowering time in Arabidopsis. Plant Physiol., 159:239-50.

Li Y.X., Zhang W., Dong H.W.,Liu Z.H., Ma J. and Zhang X.Y. (2018). Salicylic acid in Populus tomentosa is a remote signalling molecule induced by Botryosphaeria dothidea infection. Sci. Rep. 8: 14059. https://doi.org/10.1038/s41598-01832204-9.

Li N., Han X., Feng D., Yuan G. and Huang, L.H. (2019a). Signaling Crosstalk between Salicylic Acid and Ethylene/Jasmonate in Plant Defense: Do We Understand What They Are Whispering?. Int. J. Mol. Sci., 20: 671; doi:10.3390/ijms20030671

Li T., Huang Y., Xu Z., Wang F. and Xiong A.S. (2019b) Salicylic acid-induced differential resistance to the Tomato yellow leaf curl virus among resistant and susceptible tomato cultivars. BMC. Plant. Biol., $\quad 19$ : 173. https://doi.org/10.1186/s12870-019-17840

Liu L., Sonbol F.M., Huot B., Gu Y., Withers J., Mwimba M.,Yao J., He S.Y. and Dong X. (2016). Salicylic acid receptors activate jasmonic acid signalling through a non-canonical pathway to promote effector-triggered immunity. Nat. Commun., 7: 13099.

Liu L., Zhang J., Adrian J., Gissot L., Coupland G., Yu D. and Turck F. (2014). Elevated levels of MYB30 in the phloem accelerate flowering in Arabidopsis through the regulation of FLOWERING
LOCUS T. PLoS One., 9:e89799. doi:10.1371/journal.pone.0089799.

Liu X., Kristin S., Rockett, Camilla J. Kørner and Karolina M. Pajerowska-Mukhtar. (2015). Salicylic acid signalling: new insights and prospects at a quarter-century milestone. Essays Biochem., 58: 101-113: doi: 10.1042/BSE0580101

Lucas J.A., Hawkins N.J.and Fraaije B.A.(2015). The evolution of fungicide resistance. Adv Appl Microbiol., 90:2992.

Malamy J., Carr J.P., Klessig D.F. and Raskin I. (1990). Salicylic acid: a likely endogenous signal in the resistance response of tobacco to viral infection. Science. 250:1002-4.

Mauch F., MauchMani B., Gaille C., Kull B., Haas D., and Reimmann C. (2001) Manipulation of salicylate content in Arabidopsis thaliana by the expression of an engineered bacterial salicylate synthase. Plant. J. 25:67-77.doi: 10.1046/j.1365-313x.2001.00940.x

Métraux J.P., Signer H., Ryals J., Ward E., Wyss-Benz M., Gaudin J., Raschdorf K., Schmid E., Blum W. and Inverardi B.(1990). Increase in salicylic acid at the onset of systemic acquired resistance in cucumber. Science., 250:1004-6.

Mur L.A.J., Kenton P., Atzorn R., Miersch O. and Wasternack C. (2006). The Outcomes of Concentration-Specific Interactions between Salicylate and Jasmonate Signaling Include Synergy, Antagonism and Oxidative Stress Leading to Cell Death. Plant Physiol., 140: 249-262.

Norman C., Howell K.A., Millar A.H., Whelan J.M. and Day D.A. (2004). Salicylic acid is an uncoupler and inhibitor of mitochondrial electron transport. Plant Physiol., 134:492-501.

Pajerowska-Mukhtar K.M., Wang W., Tada Y., Oka N., Tucker C.L. and Fonseca J. P. (2012). The HSF-like transcription factor TBF1 is a major molecular switch for plant growth-to-defense transition. Curr. Biol., 22: 103-112.doi: 10.1016/j.cub.2011.12.015 
Pallas J.A., Paiva N.L., Lamb C. and Dixon R.A. (1996). Tobacco plants epigenetically suppressed in phenylalanine ammonia-lyase expression do not develop systemic acquired resistance in response to infection by tobacco mosaic virus. Plant J., 10:281-93.

Raskin I. (1992). Role of salicylic acid in plants. Annu Rev Plant Physiol., 43:43963.

Rhoads D.M. and McIntosh L. (1992). Salicylic acid regulation of respiration in higher plants: alternative oxidase expression. Plant Cell., 4:1131-9.

Robert-Seilaniantz A., Grant M. and Jones J.D.G. (2011). Hormone crosstalk in plant disease and defense: more than just JASMONATE-SALICYLATE

antagonism. Annu. Rev. Phytopathol., 49:317-43.

Sadeghi M.,Dehghan S.,Fischer R.,Wenzel U.,Vilcinskas A. and Kavousi, H. R. (2013). Isolation and characterization of isochorismate synthase and cinnamate 4hydroxylase during salinity stress, wounding and salicylic acid treatment in Carthamus tinctorius. Plant. Signal. Behav., 8:e27335. doi:10.4161/psb.27335

Sánchez G., Gerhardt N., Siciliano F., Vojnov A., Malcuit I. and Marano M.R. (2010). Salicylic acid is involved in the $\mathrm{Nb}$ mediated defense responses to Potato virus X in Solanum tuberosum. Mol. Plant. Microbe. Interact., 23:394-405.

Schreinemachers P. and Tipraqsa P. (2012). Agricultural pesticides and land use intensification in high, middle and low income countries. Food Policy., 37:61626.

Seyfferth C. and Tsuda K. (2014). Salicylic acid signal transduction: the initiation of biosynthesis, perception and transcriptional reprogramming. Front. Plant. Sci., 5:697. doi:10.3389/fpls.2014.00697.

Singh V., Roy S., Giri, M.K., Chaturvedi R., Chowdhury Z., Shah J. and Nandi A.K. (2013). Arabidopsis thaliana FLOWERING LOCUS D is required for systemic acquired resistance. Mol. Plant. Microbe. Interact., 26:1079-88.

Spoel S.H. and Dong X. (2012) How do plants achieve immunity? Defence without specialized immune cells. Nat Rev Immunol., 12:89-100.

Stael S., Kmiecik P., Willems P., Van Der Kelen K., Coll N.S., Teige M. and Van Breusegem F. (2015). Plant innate immunity-sunny side up? Trends Plant Sci., 20:3-11.

Strawn M.A., Marr S.K., Inoue K., Inada N., Zubieta C., and Wildermuth M.C. (2007). Arabidopsis isochorismate synthase functional in pathogen-induced salicylate biosynthesis exhibits properties consistent with a role in diverse stress responses. $J$. Biol.Chem., 282: 59195933.doi:10.1074/jbc.M605193200.

Thomma B.P.H.J., Nürnberger T. and Joosten M.H.A.J. (2011). Of PAMPS and effectors: the blurred PTI-ETI dichotomy. Plant. Cell., 23:4-15.

Tripathi D., Raikhy G. and Kumar D. (2019). Chemical elicitors of systemic acquired resistance-Salicylic acid and its functional analogs. Curr. Plant. Biol., 17: 48-59.

Tsuchiya T. and Eulgem T. (2010). The Arabidopsis defense component EDM2 affects the floral transition in an FLCdependent manner. Plant J., 62:518-28.

Uppalapati S.R., Ishiga Y., Wangdi T., Kunkel B.N., Anand A. and Mysore K.S. (2007). The phytotoxin coronatine contributes to pathogen fitness and is required for suppression of salicylic acid accumulation in tomato inoculated with Pseudomonas syringae pv. tomato DC3000. Mol. Plant. Microbe. Interact., 20: 955-965. doi:10.1094/MPMI-20-8-0955

Vallad G.E. and Goodman R.M. (2004). Systemic acquired resistance and induced systemic resistance in conventional agriculture. Crop. Sci., 44:1920-34.

van Hulten M., Pelser M., van Loon L.C., Pieterse C.M.J. and Ton J. (2006). Costs and benefits of priming for defense in Arabidopsis. Proc. Natl. Acad. Sci. US A., 103:5602-7. 
Verberne M.C., Verpoorte R., Bol J.F., Mercado-Blanco J. and Linthorst H. J. (2000). Overproduction of salicylic acid in plants by bacterial transgenes enhances pathogen resistance. Nat. Biotechnol., 18: 779-783.doi:10.1038/77347

Vernooij B., Friedrich L., Morse A., Reist R., Kolditz-Jawhar R., Ward E., Uknes S., Kessmann H. and Ryals J.(1994). Salicylic acid is not the translocated signal responsible for inducing systemic acquired resistance but is required in signal transduction. Plant Cell., 6:959-65.

Vlot A.C., Dempsey D.A. and Klessig D.F. (2009). Salicylic acid, a multifaceted hormone to combat disease. Annu. Rev. Phytopathol., 47:177-206.

Walters D.R., Ratsep J. and Havis N.D. (2013). Controlling crop diseases using induced resistance: challenges for the future. $J$. Exp. Bot., 64:1263-80.

Wang G.F., Seabolt S., Hamdoun S., Ng G., Park J. and Lu H. (2011). Multiple roles of WIN3 in regulating disease resistance, cell death, and flowering time in Arabidopsis. Plant Physiol., 156:1508-19.

Weissman G. (1991). Aspirin. Sci. Amer., 264:84-90.

White R.F. (1979). Acetyl salicylic acid induces resistance to tobacco mosaic virus in tobacco. Virology., 99: 410-412.
Wildermuth M.C., Dewdney J., Wu G. and Ausubel F.M. (2001). Isochorismate synthase is required to synthesize salicylic acid for plant defence. Nature., 414: 562-565. doi:10.1038/35107108

Yang Y., Chen T., Ling X. and Ma Z. 2017. Gbvdr6, a Gene Encoding a ReceptorLike Protein of Cotton (Gossypium barbadense), Confers Resistance to Verticillium Wilt in Arabidopsis and Upland Cotton. Front Plant Sci., 8: 2272. doi: 10.3389/fpls.2017.02272.

Yoshioka K., Nakashita H., Klessig D.F. and Yamaguchi I. (2001) Probenazole induces systemic acquired resistance in Arabidopsis with a novel type of action. Plant. J., 25:149-57.

YuanY.,Chung J.D., Fu X., Johnson V.E., Ranjan P. and Booth S.L. (2009). Alternative splicing and gene duplication differentially shaped the regulation of Isochorismate synthase in Populus and Arabidopsis. Proc. Natl. Acad. Sci. U.S.A. 106: 22020-22025. doi:10.1073/pnas.0906869106

Zhu F., Xi D.H., Yuan S., Xu F., Zhang D.W. and Lin H.H. (2014). Salicylic acid and jasmonic acid are essential for systemic resistance against tobacco mosaic virus in Nicotiana benthamiana. Mol. Plant. Microbe. Interact., 27(6):567-77.

\section{How to cite this article:}

Valarmathi, P. 2020. Salicylic Acid, A Versatile Hormone to Combat Diseases in Cotton. Int.J.Curr.Microbiol.App.Sci. 9(07): 33-47. doi: https://doi.org/10.20546/ijcmas.2020.907.005 